\title{
Investigation of Network-Based Information System Model
}

\author{
A.M. Konrad, M. Perez, J. Rivera, Y. Rodriguez, \\ M.J. Durst, D.W. Merrill, and H.H. Holmes \\ Information and Computing \\ Sciences Division
}

September 1996 


\section{DISCLAIMER}

This document was prepared as an account of work sponsored by the United States Government. While this document is believed to contain correct information, neither the United States Government nor any agency thereof, nor The Regents of the University of California, nor any of their employees, makes any warranty, express or implied, or assumes any legal responsibility for the accuracy, completeness, or usefulness of any information, apparatus, product, or process disclosed, or represents that its use would not infringe privately owned rights. Reference herein to any specific commercial product, process, or service by its trade name, trademark, manufacturer, or otherwise, does not necessarily constitute or imply its endorsement, recommendation, or favoring by the United States Government or any agency thereof, or The Regents of the University of California. The views and opinions of authors expressed herein do not necessarily state or reflect those of the United States Government or any agency thereof, or The Regents of the University of Califomia.

Available to DOE and DOE Contractors from the Office of Scientific and Technical Information

P.O. Box 62, Oak Ridge, TN 37831

Prices available from (615) 576-8401

Available to the public from the

National Technical Information Service

U.S. Department of Commerce

5285 Port Royal Road, Springfield, VA 22161

Emest Orlando Lawrence Berkeley National Laboratory is an equal opportunity employer. 
LBNL-39163

UC-600

\title{
Investigation of Network-Based Information System Model
}

\author{
A.M. Konrad, M. Perez, J. Rivera, Y. Rodriguez, \\ M.J. Durst, D.W. Merrill, and H.H. Holmes \\ Information and Computing Sciences Division \\ Ernest Orlando Lawrence Berkeley National Laboratory \\ University of California \\ Berkeley, California 94720
}

September 1996

This work was supported by the Assistant Secretary for Environment, Safety and Health, of the U.S. Department of Energy under Contract No. DE-AC03-76SF00098. 



\section{DISCLAIMER}

Portions of this document may be illegible in electronic image products. Images are produced from the best available original document. 


\section{Table of Contents}

Acknowledgments

Abstract

1. Statement of problem

2. Approach

3. Architectural Issues

4. System evaluation

5. Prototype Implementation

6. Results

7. Significance of this Work

8. Possible Future Work

9. Summary of Deliverables

APPENDDX A APPENDIX B APPENDIX $C$ APPENDIX D APPENDIX E
DBMS Evaluation Criteria, detail Products and Systems Evaluated Table of Evaluations (interim) Selected Screens from WWW Access to CEDR Structured Documentation

Selected Screens from WWW Access to Epidemiology Guides (site archive records) 


\section{Acknowledgments}

This project was supported by the LBNL Center for Science and Engineering Education (CSEE). We acknowledge the support of Laurel Egenberger and Nancy Salee of CSEE. We acknowledge the support of Barbara Brooks, Heather Stockwell, and Patrick Heinig with the office of the Assistant Secretary for Environment, Safety and Health in the U.S. Department. of Energy, Environment and Health and Safety, who provide support for the CEDR Information System at LBNL. Guidance from colleagues at SLAC and LBNL was essential in concluding a successful Summer Research Program. We thank Louise Addis, George Crane, Dick Guertin (Stanford/RLIN), Pat Kreitz, and John Halperin, for their support from SLAC and Stanford, and John McCarthy and Mark Dedlow from Berkeley. 


\begin{abstract}
The objective of the DOE-LBNL summer student research program in computer and information sciences focused on investigating database-based http-based information architectures, and implementation of a prototype using DOE's Comprehensive Epidemiologic Data Resource (CEDR) metadata or Epidemiology Guide content. We were successful in identifying the components of such an information system, an appropriate configuration given the requirements, and in implementing a prototype. This work comprised investigation of various information system architectures or variants, evaluation and selection of various tools, products, and packages, preparation of databases, database content, output formats, and graphical (World Wide Web-compatible) interfaces. We successfully prepared and demonstrated network access to content from both the CEDR structured documentation and from the DOE Epidemiology Guides (site archive records).
\end{abstract}




\section{Statement of problem}

The Information Problem, how to connect people with information, is complex, in part because there are many types of obstacles and intermediaries to mitigate those obstacles. The widespread use of network-based database technologies, including the internet, the world wide web (WWW), browsers, and server-to-database interfaces (Common Gateway Interfaces, CGI) have solved a number of access problems which confront the information seeker, while requiring information system designers to become familiar with a continuous stream of new architectures, tools, and methods.

During the course of this summer, Madel Perez, Yamilet Rodriguez and Jose Rivera worked under the supervision of Allan Konrad and Mark Durst with the Comprehensive Epidemiologic Data Resource (CEDR) program to investigate new information system architectures for CEDR. CEDR is a Department Of Energy (DOE) program to disseminate by various means, including electronic, access to research results and data from DOErelated epidemiologic studies during the last 30 years.

The tasks for this Summer Research Program were to develop a set of criteria for WWWbased access to the CEDR structured documentation (metadata) database, then an architecture, then determine what components were required, and of those, which could be obtained externally and which had to be developed $a b$ initio. First it was necessary to evaluate a group of WWW-capable database management systems (DBMS) and select one. The time to finish all tasks for this Summer Research Program was 10 weeks. 


\section{Approach}

The following was the work plan provided to the student research assistants at the beginning of the program.

Week 1 Review of internetworking. Browsers. Review HTML command language via Yahoo.

Search Engines. Review homepage. Scan course pages.

Tasks: Individual homepage edited and mounted.

Week 2 The Research Problem: Develop model of components and functional requirements for Webware. Understand data flows. Review of information retrieval (IR) functionality. CGI tools. DBMS. email servers.

Tasks: Survey candidate packages.

Presentation of plan and approach. Identify bibliographic materials as needed.

Week 3 Analysis and selection. Analysis and selection of packages and products for functionality and interoperability. Selection criteria.

Possible Applications:

Webware I (web-based groupware- email in, http in, email out, http out).

Webware II (CEDR metadata)

Webware III (web-based listserv archive)

Webware IV (CEDR catalog)

Webware V (instructional modules)

Webware VI (Epidemiologic Guides)

Tasks: Status report. Selection of component specialization by each student.

Week 4 Begin Implementation: Procurement and installation

Week 5 System Installation.

Week 6 Application implementation.

Week 7 Load demonstration data (Webware M.

Week 8 Demonstration.

Week 9 Write project reports.

Week 10 Wrap up, Poster Sessions. 


\section{Architectural Issues}

At the beginning of the project, we envisaged an architecture consisting of a web server (httpd), a database management system (DBMS) with several CEDR databases, and one or more Common Gateway Interface (CGI) scripts to convey data between the httpd and DBMS and further transform the data as needed in each direction. We anticipated as a major subtask of the summer project, writing these CGI scripts ourselves, using the PERL language.

Early into the project, we learned from LBNL colleagues and other sources, that this "home brew" approach to CGI scripts was only the first step in an evolution. The CGI Evolution, $P$ hase I below indicates the originally anticipated configuration.

\subsection{CGI Evolution, Phase I}

By the "home brew" approach, we mean:

the hand-crafting of a CGI script specifically for the target database, i.e., with specific business logic regarding data base name, database elements, and searchable indices;

and where the systemic operation is:

request by client of a graphical interface form from server for specification of inquiry specifications;

generation of GET/POST requests to be sent to httpd from client;

requests received from client by httpd translated into CGI variables or stdin;

requests received by CGI script as variables or stdin, reformatted by the CGI script as legal DBMS commands;

requests received by the DBMS, processed against the database, results generated by the database, and then returned to CGI script;

results received by CGI script, translated, with substantial business logic encoding, into HTML, and forwarded to the httpd;

httpd conveys html using http to client browser. 
A block diagram, figure 1, illustrates this architecture, comprised of the following:

Client:

C1 Platform (hardware, OS, network connectivity)

C2 Browser (http-capable if not multi-protocol)

Network:

N1 Network

Server:

S1 Platform (hardware, OS, network connectivity)

S2 HTTP Daemon

S3 Page containing a Link (optional, but likely)

S4 Form Page

S5 CGI script

S6 DBMS

S7 HLI/means of send/receive data to other processes (If on same machine*)

*I.e., if $\mathbf{S 6}$ is on a different network address (i.e., different machine) than S5, then S7 becomes a network server, to mitigate the network boundary, and listens at a (well known) port, and binds an incoming socket to the port to support a specific client process.

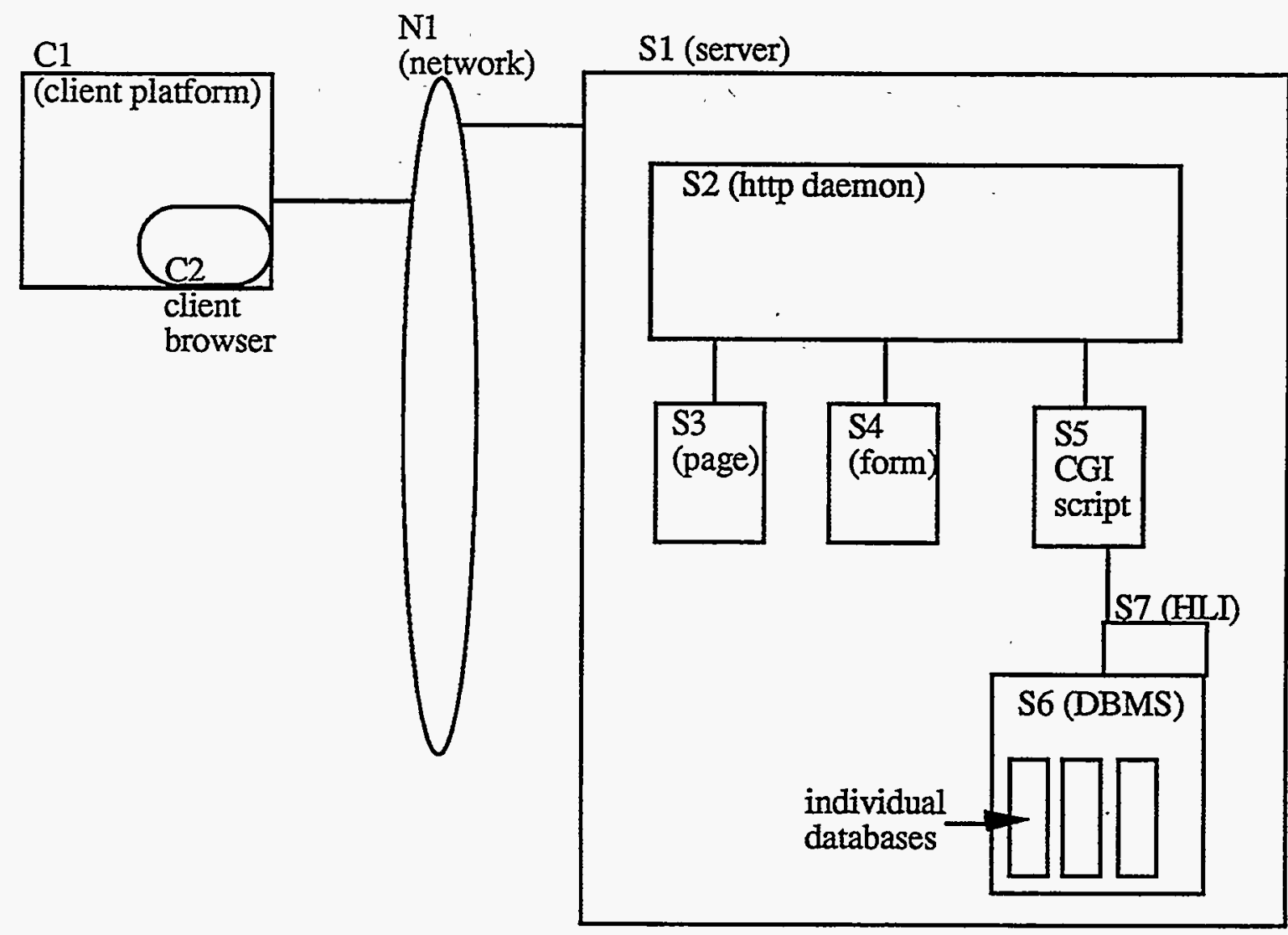

Figure 1 
The components provide the following services:

C1 human interface service and network connectivity

C2 http communications capability, HTML rendering capability, 'helper' applications

$\mathrm{N} 1 \quad \mathrm{TCP} / \mathrm{PP}$

S1 All of: HTTPD service, DBMS service, and network connectivity

S2 Send and Receive http commands (as/from HTML documents) to/from Client, translate commands, and send to/from CGI Script

S3 Store HTML text and URL pointing to a forms page

S4 Provide forms capabilities (fields, radio buttons, etc.) to specify a search or browse, and return values to HTTPD

S5 Both:

Receives forms input, translates into DBMS-acceptable languages, conveys to DBMS;

Receives DBMS response, performs HTML markup, conveys to Client Browser (C2) via S2.

Note: S5 might run on a machine with a different network address than httpd (S2) and thus be supported as a CGI program encapsulated in a network server, listening for connection requests, queuing, etc.

S6 DBMS capability (organized repository, indexing, input, output/report generator, query processing)

S7 Interoperability with DBMS by process other than a terminal, .e.g, a CGI script DBMS same machine as CGI: Host Language Interface DBMS different machine as CGI: server listening at a port 
How Capability is Provided (Interoperabilitv):

Pertinent interoperations are:

C2-S2 http, ftp, gopher, etc.

S2-S3 HTML, etc.

S2-S4 GET (constructs a URL)

POST (constructs MIME object)

S2-S5 (S4-S5) CGI recognition defined for httpd functionality

To CGI: GET/URL stores variables in memory accessible to CGI POST/MIME sends to CGI via stdin

From CGI: stdout

S5-S6/S7 To DBMS: Same Machine: HLI.

Different Machine: network server

From DBMS: Same Machine: HLI.

Different Machine: network server

Does Application Developer Provide the Component?

C1 No, User

C2 No, User

N1 No, System

S1 No, System

S2 No, System

S3 Yes, we develop

S4 Yes, we develop

S5 Yes, we develop

S6 No, Vendor

S7 No, Vendor 


\subsection{CGI Evolution, Phase II}

The essence of this phase is that laborious hand-crafting of CGI middleware to provide the services for S5 supra is virtually eliminated by redistributing the functionality of S5 (CGI script) as follows:

S5 still receives requests from httpd and passes to DBMS, and receives requests from DBMS and returns to CGI

However, most translation tasks and other database-specific tasks, e.g., translating forms/CGI requests into DBMS requests, and translating DBMS responses into HTML objects, are no longer performed by the CGI, but are partitioned to either the httpd, in the case of translating forms requests into dbms-legal commands), or assigned to the DBMS itself (generating HTML-ready output).

This implies that the httpd "knows" the syntax of DBMS-legal requests. This is accomplished by 'built-in' recognition of translations between forms specifications and legal DBMS command language, and facilitated by using a configuration file which is consulted by the httpd where application-specific attributes are specified.

Indeed, major database vendors are bringing to market their own httpd servers which provide these translation services without need for individual coding of CGI scripts. The CGI functionality of conveying requests in each direction is reduced to a generic capability that can be coded and compiled by the httpd/DBMS vendor as simply another module.

A Phase II block diagram (figure 2) illustrates this modified architecture. This model comprises additional components:

S8 config files containing application-specific information such as name of database, user, passwords, preference for error processing, logging, format names, other specifications as required.

S9 Stored procedure call processor accepts requests from httpd as configured according to individual config file specifications and transmitted by the CGI, and submits the query to the specified database and returns the result. This processor might be recognized to the DBMS as simply another (logged on) user , or process (if using S7 host language interface).

The benefits are:

1. The labor required to implement a forms-based www-based dbms application is greatly reduced.

2. The functionality remaining in the CGI requires no user-specific or applicationspecific information and can be treated as simply a compiled module that runs regardless of the database requested. 


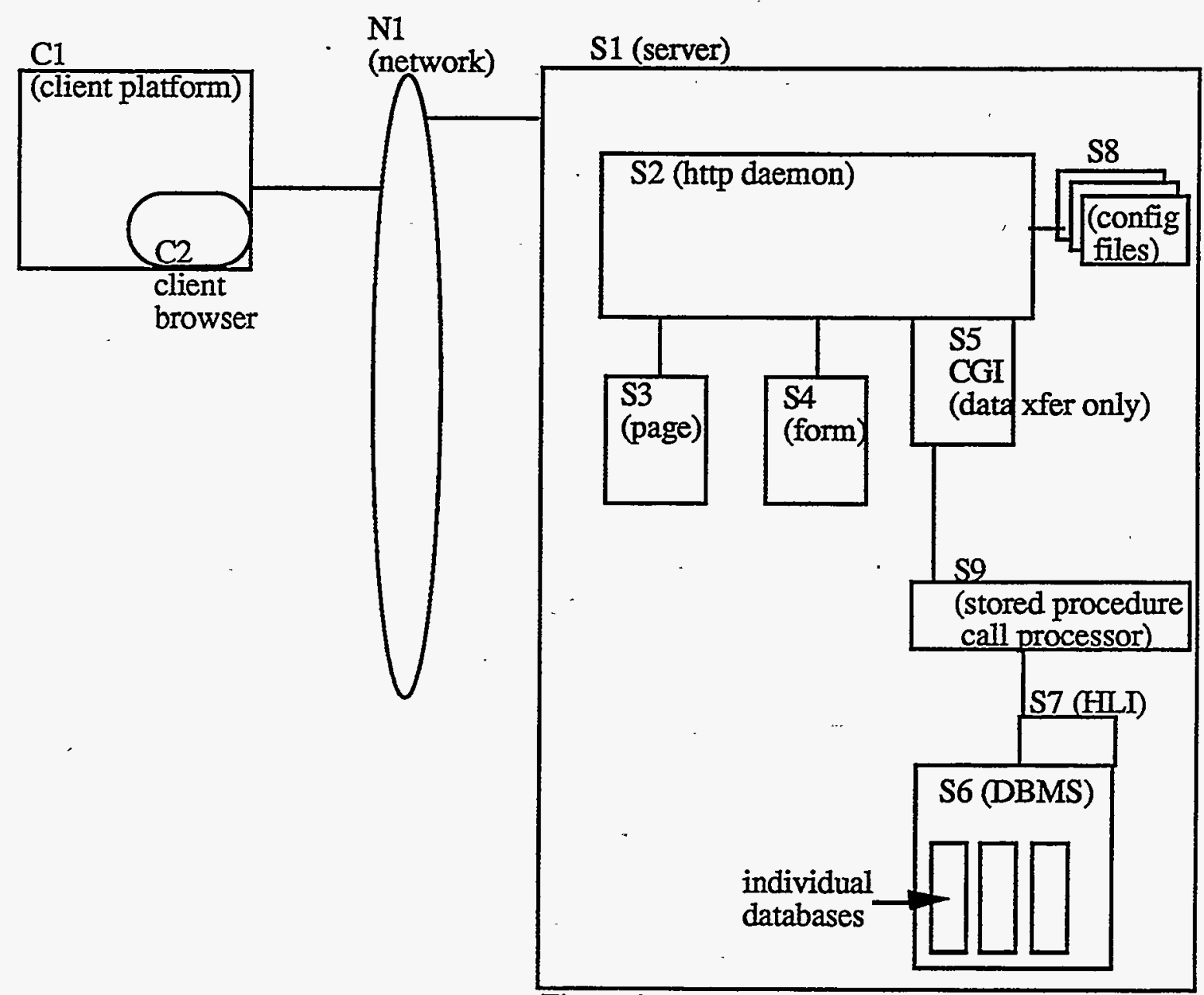

Figure 2 


\subsection{CGI Evolution, Phase III}

This phase builds upon the benefit of Phase II by establishing connectivity between S2 (httpd) and S6 (DBMS) such that each successive DBMS request does not require reinitialization of the connection between S2 and S6, and accompanying generation of an image of database access modules. Where architecture of both S2 and S6 is under the control of one party, e.g., a database vendor, this connection can be established through efficient, if proprietary, means.

The primary benefit of Phase III is enhanced performance where the overhead of establishing a connection to a database or an image of a database access module in memory is substantial or where there is a high volume of requests as a multiplier of the overhead.

\subsection{Relationship to Summer Research Project.}

In Section 4 below, is described our evaluation of various tools and systems considered for our information system architecture. During the course of our investigation, we determined:

A significant training investment is required in developing PERL expertise;

A significant coding investment is required in developing CGI scripts by hand

We have access to "Phase $\Pi^{\prime \prime}$ and "Phase II" DBMS obviating the need to take a "Phase I" approach.

As the system evaluation below indicates, we did identify a set of Phase III tools that were within our scope for (the relatively short 10-week) summer research project and purchasing constraints (time and dollars). 


\section{System and product evaluation}

The objective of the product and system evaluation effort was to identify tools with sufficient 'built-in' WWW accessibility that the overall work of implementing WWW access to CEDR databases would be minimized. Initially, we evaluated:

S5 (CGI script) tools

S6 Database Management Systems

\section{Evaluation of S5 (CGI script) tools.}

As explained supra, we learned during the course of our investigation that a Phase I approach might not be the most desirable, and that at least a Phase II approach was possible within the context of the Summer Research Program. Consequently, our initial effort devoted to developing both PERL and CGI expertise, while useful from a breadth-ofdiscipline standpoint, was abandoned in favor of a Phase II/III approach.

\section{Evaluation of S6 Database Management Systems:}

We understand database management system to comprise:

1. An organized repository of goal records stored "in" the DBMS according to a definition or schema also "in" the DBMS

2. An indexing engine, which builds indices of various kinds (word, phrase, date, personal name, etc.) from goal records newly added to (1) the organized repository; modifies index entries appropriately when goal records are modified; and deletes index entries when goal records are deleted from the organized repository.

3. A search engine which receives queries, processes them against index records to obtain search results (a set of pointers to goal records selected), and then uses those pointers to provide the count of the records satisfying the search result.

4. A report generator which uses search result sets or direct (e.g., display or sequential processing) commands to fetch specified goal records and then present them in a specified format either to a specified file, to a terminal, or to another program, such as a CGI program, for further processing or transmission to another program means.

5. Support for text/character manipulation capabilities (please see Appendix).

6. Support for concurrent update control.

7. Support for optionally-occurring elements.

8. Support for dates

9. Support for pre-defined procedures (on records upon input to organized repository, on records upon output from dbms, on goal records as passed to index records, on query values as processed against index records)

10. Support for personal name processing.

11. Support for variable length elements.

12. Support for web/http (CGI) interoperation (Phase II or Phase III) 
Systems were identified as candidates, and then evaluated against the above twelve criteria. Candidate systems were identified from a variety of sources, including:

a) WWW

1) Free Databases list from Yahoo/Database page

2) Non Free Databases - company homepages

b) FTP sites

c) Information from LBNL colleagues

d) Information from external colleagues

From these sources, a preliminary list of candidate systems was developed (please see Appendix B).

Evaluation was performed on each candidate system initially only to determine whether the first four criteria were met by the candidate system. This resulted in a narrower list of finalists for which further evaluation was performed using criteria $5-12$.

Evaluation on any candidate system was done by whatever means were available to us within the constraints of our Summer Research Program. We did not invite any vendor for a presentation. We began evaluation of nearly every candidate with information from the vendor or distributor's web pages, ftp sites, or other information we were able to obtain. We utilized:
1) Telephone
2) E-mail
3) Fax
4) Regular Mail
5) Web sites
4) FTP sites 
Results of Evaluation.

Twenty two (22) systems received preliminary evaluation. Of these:

Seven (7) were "web search engines only". That is, they did not support goal records in an organized repository of their own, but used agents to scan the web, fetch documents, and build word indices. These were considered unsuitable because, without an organized repository and a record structure under CEDR's control, we could not control the format of output, or even generate reformatted output at all. These were:
1) Glimpse 3.0
2) lq-text
3) FFW-Free text for WWW
4) MG Information Retrieval System
5) qt (Query Text)
6) Topic Internet Server
7) WAIS

Four (4) were non-responsive. In this category, we include those for which no telephone number or network address was found for obtaining further information, or for which the distributor indicated there was 'no support available' for the product, or where repeated telephone inquiries were not returned, or where our inquiries were answered with "the only documentation we have comes with our system - get our system and install it, and then you can read the documentation". These included:
1) Postgres 95
2) MORE-Multimedia Oriented Repository Environment
3) System II
4) SMART

More detailed evaluation was performed upon the eleven (11) remaining systems, with the following results:

There were several products which did not meet functional requirements related to bibliographic-type applications (criteria 5-11):
1) $\mathrm{RDB}$
2) ORACLE (base product)
3) Sybase (base product)

Sybase and ORACLE both recommended use of separate ancillary products to meet the bibliographic/textual requirements. We read these recommendation to mean that the core DBMS offerings themselves were not adequate to meet our requirements in the judgement of the vendors. One of these ancillaries was a third-party product, one was a product offering of the primary DBMS manufacturer. Although the combination of these DBMS products and their application add-ons might have met our functional requirements, we discontinued further evaluation because the investments required, both in licensing and in staff training time, were beyond the scope of our Summer Research Program. If an evaluation process less constrained by time and other resources becomes possible, these two, Oracle with Context, and Sybase with Fulcrum, might be given further consideration.

There was a class of products which can be described as WWW-DBMS middleware. Although these did not meet our DBMS evaluation criteria, they could provide an interesting alternative approach in a less constraining evaluation program. We encountered one such product (although we encountered mention of a small number of others):

1) ESQL/Seekersoft 
One system was not evaluated because we determined that it was still in beta stage on the basis of postings to a listserv established for users and beta testers. However, a future evaluation should include this system:

1) ISITE/ISEARCH from Center for Networked Information Discovery and Retrieval (CNIDR)

The remaining six (6) appeared to be finalist candidates:
1) SPIRES
2) AIRS II
3) BRS/Search
4) Microsoft Access
5) Basis Plus
6) Fulcrum Search Server

Each of these systems or products deserve full evaluation in the context of a conventional evaluation process.

By this point in our Summer Research Program, having identified these six from a field of twenty-two, we were confronted with the pressure of selecting and installing a product, loading CEDR data, and developing WWW interface forms, all within about six (6) weeks and with negligible funding or time resources to conduct any sort of convention procurement.

At this juncture, we received indications form a sister DOE laboratory, the Stanford Linear Accelerator Center (SLAC) near Palo Alto, of interest in inviting our Summer Research Project to use one of the six finalists candidates, SPIRES, using their computing resources, at no cost and with no procurement burden. Further, this alternative offered a Phase III approach, thus mitigating resource-intensive CGI development, a comparable computing platform to CEDR's (SunOS 4.1.3), and favorable licensing terms if migration to an LBNL platform were desired. Interestingly, SPIRES provides this Phase III capability, the performance enhancement of optimized reinitialization, without a proprietary httpd, but by instantiating the initialization functionality in S9 rather than in S2.

SPIRES, developed with DOE funding and the foundation for a large (DOE ER/SLAC/CERN/DESY/Japan) high energy physics bibliographic database, is recognized by portions of DOE (ER) as an appropriate tool for this type of application.

Finally, this alternative had the advantage that a project staff member had sufficient prior working knowledge of the system that there was a high probability that we could define CEDR databases, load CEDR data, and develop WWW interfaces within the tight time constraints. Additionally, we had established a good working relationship with SLAC staff over the past two decades which augured for good technical support when needed by the Summer Research Project staff.

SLAC's motivation for this offer was consistent with other cooperative work between SLAC and LBNL in the past: mutual benefit. SLAC was interested in our experience with developing WWW-based applications in SPIRES which they might use to improve the transition of their mainframe-based SPIRES high energy physics databases to a unix-based www-based environment.

Consequently, SPIRES was chosen from the six finalist candidate systems with which to implement our prototype system for the Summer Research Project. 


\section{Prototype Implementation}

The prototype information system implemented in the Summer Research Project was a Phase III type information system as described above.

Three variations to the Phase III model are used in the WWW-based SPIRES system:

First, noted supra, the performance enhancement of optimized reinitialization is achieved without a proprietary httpd by instantiating the initialization functionality in S9 (stored procedure processor) rather than in S2 (httpd) or S5 (CGI).

Second, use of a proprietary httpd is further avoided by provision of a proprietary module which provides forms-DBMS request translation unique to SPIRES. This appears in figure 3 as component S10, DBMS-forms Intèrface.

Third, the network address of the httpd server (S2) and the network address of the machine running the CGI script (S5) are different, i.e., they are different machines. Consequently, the CGI function is encapsulated in a server (S5) which listens in the standard way for requests from S2, otherwise behaving as an ordinary Phase II CGI component (passing requests only), although some additional security features have been implemented in this CGI server.

The following table indicates the names used in the CEDR WWW-based SPIRES information system for each of the generic components:

\section{Component Generic Name CEDR-WWW-SPIRES}

S1 Platform

S2

S3

S4

S5

S6

S8
HTTP Daemon

Page containing link to search form

Form Page

CGI function

(Phase II type)

DBMS

config files
SunOS Release 4.1.3_U1 (SERVER-4M)

(all versions of unix believed to be supportable)

Version 3.0pre5 of the CERN server code is running on www.slac.stanford.edu (the Unix production server).

not public at present

http://www.slac.stanford.edu/ -1b15spi/formcedr.html http://www.slac.stanford.edu/ lbl5spi/formepi.html

encapsulated in John Halperin CGI-SPIRES server $\sim j x h / p g m s / s p i r e s / j r u n s r v r . c s h$ (netcall.pl) (interpreted rather than compiled at present)

SPIRES, uSPIRES (unix).

stored in: /afs/slac.stanford.edu/www/spires/find/ cedrdfs cedrfile cedruars cedrcode

When spiface receives the request from a form, then it consults the options files for that database, reforms the command to be a 'qspires' command and sends it on to Halperin's server. 
Stored procedure - quSPIRES (a version of qSPIRES for unix) call processor

S10 DBMS-Forms interface

SPIFACE v. 2.0

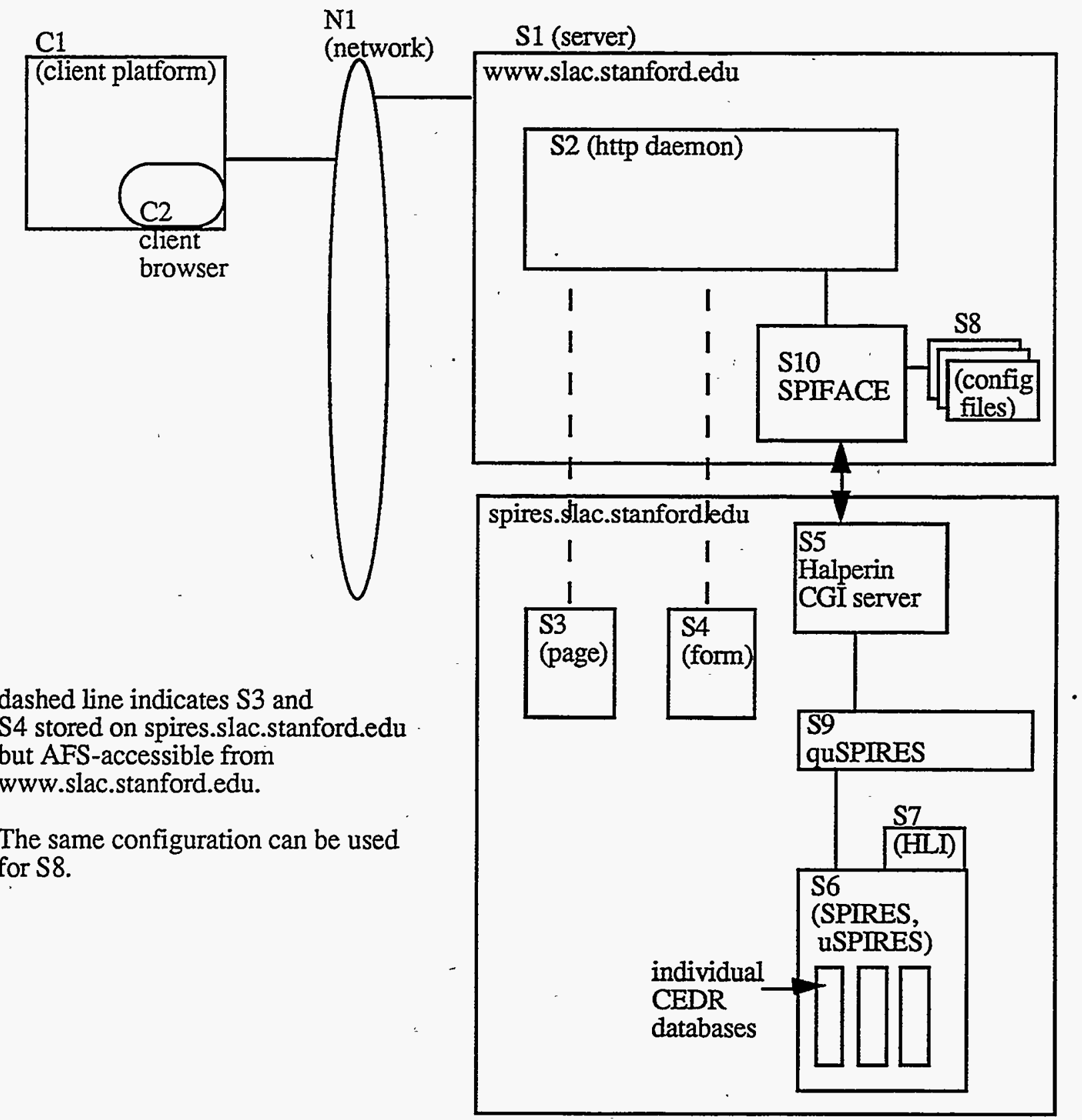

Figure 3 


\section{Results}

1. We defined a file definition (schema) for the Epidemiology Guides comprising two 'databases':

\section{Epiguides}

EpiDE (the 'data elements' defined by HAI - a lookup table)

2. We converted three of the eight Epi Guides for loading, and loaded.

3. We developed prototype output formats, including for web presentation.

4. We implemented www-accessible forms for access to the Epidemiology Guides, for browsing, and for searching.

http://www.slac.stanford.edu/ lbl5spi/formepi.html

5. We defined a file definition (schema) for the CEDR metadata comprising five 'databases':

CEDRDFS (Data file set level metadata)

CEDRFILE (File level metadata)

CEDRVARS (Variable level metadata)

CEDRCODE (Codes metadata)

CEDRICD (Death Summaries)

6. We developed conversion routines to convert each type of metadata from CEDR dump format into database load format. This was problematic owing to the inconsistency of CEDR data and some of the CEDR dumping routines, and other eccentricities inherent in the current CEDR.

7. We converted all CEDR metadata resident in cedr.lbl.gov/data/cedrprod as of midJuly.

8. We loaded all the CEDR metadata and ICD summaries into their respective databases.

9. We established output formats for each metadata database, including for www access.

10. We established configuration files and www-accessible forms for access to all five databases.

http://www.slac.stanford.edu/ lbl5spi/formcedr.html

11. We itemized a number of aspects of CEDR data deserving of QA/QC attention.

12. We characterized potential future work (Section 8). 


\section{Significance of this Work}

All network access to CEDR material previously was to stored "flat files" each of which had to be separately maintained.

With this prototype, interaction with CEDR structured documentation is directly to the authoritative data in the database, giving these benefits:

1. The laborious load-dump-filter/overflow file procedure can eventually be abandoned.

2. Updated CEDR information is now accessible instantly- when the dbms record is updated, it is available on the on the web at that moment.

3. Multiple output formats all operate on single source - when CEDR metadata is updated, it can be instantly accessible in www format, CEDRtext format, and in phototypesetter format.

4. The HyperCEDRtext concept is now obsolete. 


\section{Possible Future Work}

Transforming the Summer Research Project prototype to a production CEDR information system might require at least:

1. Additional output formats to generate CEDRtext files.

2. Development of a protocol to enable publish-on-demand CEDR catalogs.

An important aspect of this work is that a CEDR staff member can update a metadata record, and the update is immediately available, and could be used to print a catalog rapidly. Such on-demand catalog printing might be triggered by receipt of email with a valid postal address.

3. Development of a CEDRtext applet:

Currently, the only functional benefit of CEDRtext over www access is that CEDRtext only sends to the user/terminal that portion of the numerical data (i.e., data, not metadata) that fits in a small (15x80) window. Were the CEDR data files to be simply accessed as HTML, the larger CEDR files would exceed the capacity of the net and the client's browser.

However, a Java-like applet might be developed with this portion of CEDRtext functionality, and would work as follows:

- Information Seeker opens CEDR homepage

- (registered) Information Seeker clicks on link to CEDR data

- A CEDR applet is conveyed from server to client's browser

-The applet provides a window with vertical and horizontal scroll bars.

Manipulation of the scroll bars causes command language on the server to fetch and send to the client only those records that fit, thus avoiding the problem of sending whole CEDR files to the client.

4. Complete installation of the remaining Epi Guides.

5. Aesthetics. The on-screen presentation of both CEDR structured documentation and epidemiology guides can be improved.

6. Include HTML links in CEDR metadata to other metadata (Epiguide goal records 'data element' element now linked to the 'Epi data element' database).

7. Complete loading of all CEDR structured documentation.

8. Webware I (web-based groupware- email in, http in, email out, http out).

9. Webware III (web-based listserv archive)

10. Webware $\mathrm{V}$ (instructional modules) 


\section{Summary of Deliverables}

Online Deliverables:

1. Epi Guides: http://www.slac.stanford.edu/ lbl5spi/formepi.html

2. CEDR Metadata: http://www.slac.stanford.edu/ lbl5spi/formcedr.html

\section{Paper Deliverables:}

1. LBNL-39163 Investigation of Network-Based Information System Model,

Konrad, Perez, Rodriguez, Rivera, Durst, Merrill, Holmes. September, 1996 (this report). 


\section{Appendix A}

\section{DBMS System Evaluation Criteria}

Our first four general requirements are:

a) Organized Repository of Goal Records

b) Generation and maintenance of Index Records derived from Goal Records

c) Query of Index Records

d) Presentation of Goal Records both by search result or directly (Report Generator)

Additionally, the following requirements relate to bibliographic database applications:

a) Text/Character manipulation capabilities.

1) Breaking string into words for passing to index on query string.

2) Force to upper case for passing to index and on query string.

3) Change a string to different string.

4) Insert text in a string.

5) Return size of data element value.

6) Squeeze leading and trailing blanks.

b) Concurrent update control.

c) Optionally-occurring elements

1)The data elements(variables) use storage or cells having no value?

d) Dates.

1)What's the DATE FORMAT?

2)Is it possible to change the DATE in one format to different output format?

e) Pre-defined procedures

1)What Pre-defined procedures process values as input to DB?

2)What Pre-defined procedures process output or displayed values from DB?

3)What Pre-defined procedures process index values?

4)What Pre-defined procedures process search values?

f) Personal name processing.

1)How does DB store personal names?

2) How does $D B$ retrieve personal names?

3)How does DB process and present personal names sub-components?

g) Variable length elements.

1)Can all data elements in a DB be variable length?

2) How is storage of variable length values supported?

h) Data repository.

1)Is the data stored in the DB or externally?

1) Search engine.

1)Complexity of query language.

j) Report Generator

k) Web/http (CGD) interface.

1)Description of DBMS for interoperability with CGI scripts. 



\section{Appendix B}

\section{Evaluation of products and systems}

\section{Microsoft Access}

Name of the manufacturer: Microsoft Corporation

Name of the vendor: Microsoft Corporation

Cost: $\$ 399.00$

Type: Relational Database

Summary: Microsoft Access 2.0 is a good DBMS for Windows, Windows 95 and Windows NT. There is Web access for

Windows NT server. No Web access was found for

Windows or Windows 95. Examples of features were

provided but no implementation details were available.

\section{Topic Internet Server}

Name of the manufacturer: Verity Inc.

Name of the vendor: Verity Inc.

Cost:

Type: Search Engine

Summary: Topic Internet Server is a product of topic SEARCH which is part of Verity's topic family of products. No detailed paper documentation was provided and there was no answer to telephone calls and mail messages.

\section{Postgres 95}

Name of the manufacturer: Andrew and Jolly Chen

Name of the vendor: University of California

Cost: Free

Type: Relational Database

Summary: Terminated evaluation at criterion number 5 (Optionally-occurring elements) on lack of merits:

1) Absence of product support

2) Absence of text capability

Resources do not permit further review.

\section{WAIS}

Name of the manufacturer: Thinking Machines, Inc.

Name of the vendor: CNIDR

Cost: Free

Type: Information retrieval system 
Summary: Free WAIS is an Information retrieval system that implements free text search using a client/server architecture. Since free WAIS is not a DBMS there is no further consideration of this product.

\section{Basis plus}

Name of the manufacturer: Information Dimension Inc.

Name of the vendor: Information Dimension Inc.

Cost:

Type: Text DBMS

Summary: BASIS plus is a great text DBMS with almost all the characteristics CEDR is looking for. The only characteristic it don't has is the personal name processing. There was no answer to the telephone call to check on the cost of the product. Further consideration of the of this product is justify. No paper documentation was provided.

\section{System II}

Name of the manufacturer: Sybase Inc.

Name of the vendor: Sybase Inc.

Cost:

Type: RDBMS

Summary: System II is a family of Database products that runs on a variety of platforms from PCs to multi-cpu super servers. Including UNIX and SunOS. Some information was received by fax, however no detailed documentation about the DB was available.

\section{SMART}

Name of the manufacturer: Gerard Salton

Name of the vendor: Cornell University

Cost: Free

Type: Not a DB, Search Engine

Summary: There is not enough documentation available, because it is necessary to install the system to get reasonable documentation. The system is only a search engine.

\section{MG Information Retrieval System}

Name of the manufacturer: I.H. Witten, A. Moffat \& T.C. Bell

Name of the vendor:

Cost: Free

Type: Not a DB, Information retrieval system

Summary: The information available is only to get the free software. To obtain documentation it is necessary to install the system or buy their new book. It is a research prototype, not a production-caliber product. Evaluation was terminated. 


\section{EFW-Free text search for WWW}

Name of the manufacturer: Multitorg project

Name of the vendor: Telenor R \& D, Norway

Cost: free for non-commercial use

Type: Not a DB, is a Search Engine

Summary: The system it's only a search engine. It is a package made to provide easy-to-use searching facilities over HTML documents. Evaluation terminated.

\section{$\underline{\text { RDB }}$}

Name of the manufacturer: Walter V. Hobbs

Name of the vendor: RAND

Cost: Free

Type: Compliant with the relational model

Summary: Text/character manipulation capabilities are not supported by RDB. PERL could be used or UNIX utilities. This is a very simple relational DBMS. I think that using this DBMS with a good search engine would be a great help. [J. Rivera]

\section{lq-text}

Name of the manufacturer: Liam R. E. Quin

Name of the vendor: University of Califormia at Berkeley

Cost: Free

Type: Not a DB. Is a search engine.

Summary: lq-text is a text retrieval engine. The system is.not what we need. Does not comprise an organized repository (DBMS requirement).

\section{at (Ouery Text)}

Name of the manufacturer: John Conover

Name of the vendor: (organization) Vixie Enterprises

Cost: Free

Type: Not a DBMS; search engine only.

Summary: The documentation sent by Mr. Conover was very good. The system is a text information retrieval system. It creates, maintains and queries a full DB. Evaluation terminated. I believe this search engine together with RDB will be a good help. [J. Rivera] 


\section{ORACLE}

Name of the manufacturer: Oracle Corp.

Name of the vendor: Richard Franceschini

Cost:

Type: Relational DB

Summary: Oracle does not support bibliography data nor text retrieval capability well within the DBMS. Oracle has a software called Context that does not require the Oracle server in the current version. The next version of Context will require the proprietary server. Context is stand-alone software that needs a program (C language) to send the information. Context has many features. Context is a natural language processing technology that identifies themes and content in English text. Evaluation terminated, because original programming effort is beyond our scope and due to procurement cost.

\section{ESQL}

Name of the manufacturer: Seekersoft

Name of the vendor: Seekersoft

Cost:

Type: WWW (CGI) interface to DBMS from HTML documents.

Summary: ESQL is a interface between HTML and a DBMS that uses

SQL. The SQL statement can be used inside the HTML code.

\section{Glimpse 3.0}

Name of the manufacturer: Udi Manber and Burra Gopal

Name of the vendor: University of Arizona and National Chung- Cheng University

Cost:

Type: Global Implicit Search (Search engine)

Summary: Glimpse is an indexing and query system that allows you to search through all your files very quickly. Glimpse supports most agrep's options including approximate matching, Boolean queries, and even some limited forms of regular expressions. Does not offer an organized data repository.

\section{AIRS II}

Name of the manufacturer: Arachnae Management Limited

Name of the vendor: Arachnae Management Limited

Cost: Server $\$ 5000$, each concurrent user/client $\$ 100$

Type: Full-text DB server

Summary: At the present, there is not enough information on this product, but it should be considered in the future for its important features that meet with some of our demands. Although the people in charge of this product were contacted by email and phone, we were not able to receive enough information as to clear up our difficulties found in the criteria evaluation. 


\section{Fulcrum Search Server}

Name of the manufacturer: Fulcrum Technologies Inc.

Name of the vendor: Fulcrum Technologies Inc.

Cost:

Type: Search Engine

Summary: Fulcrum Search Server-a high performance, multiplatform, indexing and retrieval server engine. It is your key to retrieving and publishing information across the enterprise information critical for effective decision-making. Does not support data repository.

\section{MORE- Multimedia Oriented Repository Environment}

Name of the manufacturer: David Eichman, Terry Mc Gregor and Dann Danley Name of the vendor: University of Houston

Cost: Free

Type: Metadata-based repository system

Summary: Terminated evaluation on lack of merits:

1) Absence of support

2) Absence of specific information

3) MORE is a metadata base repository- the information in its underlying DBMS isn't the objects themselves, but rather information concerning the object, which is stored using other mechanisms.

\section{Spires}

Name of the manufacturer: Stanford University

Name of the vendor: Stanford University/SLAC

Cost: Pending

Type: full bibliographic DBMS with network access

Summary: Candidate for further consideration.

\section{BRS/SEARCH/Netanswer}

Name of the manufacturer: Dataware Technologies

Name of the vendor: Dataware Technologies

Cost: $\$ 15,000$

Type: full bibliographic DBMS with network access

Summary: Candidate for further consideration.

\section{ISITE/ISEARCH}

Name of the manufacturer. Center for Networked Information Discovery and Retrieval (CNIDR)

Name of the vendor: Same

Cost: freeware

Type: still in beta 



\section{Appendix C}

\section{Table of Products Evaluated (interim)}

\begin{tabular}{|c|c|c|}
\hline Data Base & Evaluator & Evaluation Group \\
\hline FFW & Jose & a-Search Engine only \\
\hline Glimpse 3.0 & Madel & a- Search Engine only \\
\hline lq-text & Jose & a-Search Engine only \\
\hline $\mathrm{MG}$ & Jose & a-Search Engine only \\
\hline gt (Query Text) & Jose & a- Search Engine only \\
\hline SMART & Jose & a-Search Engine only \\
\hline Topic Internet Server & Yamilet & a- Search Engine only \\
\hline WAIS & Yamilet & a- Search Engine only \\
\hline ORACLE & Jose & b- Doesn't support text Processing \\
\hline ORACLE with CONTEXT & Jose & c- Resource Intensive \\
\hline MORE & Madel & d-Non Responsive \\
\hline Postgres 95 & Yamilet & d- Non Responsive \\
\hline System II & Yamilet & d- Non Responsive \\
\hline ESQL & Jose & e-RDB Support Tools \\
\hline AIRS II & Madel & f- Good Candidate \\
\hline Basis Plus & Yamilet & f- Good Candidate \\
\hline BRS/Search & Madel & f- Good Candidate \\
\hline Fulcrum Search Server & Madel & f-Good Candidate \\
\hline Microsoft Access & Yamilet & f-Good Candidate \\
\hline Spires & Madel & $\begin{array}{l}\text { f- Good Candidate } \\
\text { (Selection) }\end{array}$ \\
\hline$\overline{\mathrm{RDB}}$ & Jose & g- RDB without indexing capabilities \\
\hline
\end{tabular}



Appendix D

Selected Screens from WWW Access to CEDR Structured Documentation

D-1 


\title{
CEDR - Comprehensive Epidemiologic Data Resource
}

\author{
Information Retieval
}

\section{DFS Database:}

- Browse Data File Set Structured Documentation

- Search Data File Set Structured Documentation

\section{File Database:}

- Browse File Structured Documentation

- Search File Structured Documentation

\section{Var Database:}

- Browse Variable Structured Documentation To browse structured documentation for variables, browse via Data File Set for File level structured documentation.

- Search Variable Structured Documentation

\section{Code Database:}

- Browse Code Set Structured Documentation

- Search Code Set Structured Documentation

\section{ICD Summary Database (Death Tables):}

- Browse ICD Summaries

- Search ICD Summaries

This CEDR information retrieval facility developed by Madel Perez, Jose Rivera and Yamilet Rodriguez.

We welcome your comments and questions concerning the CEDR project, our information retrieval products and Customer Service. If you would like further information about CEDR, please contact us at:

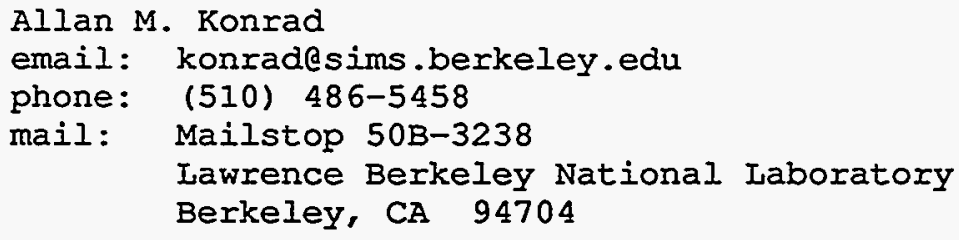




\section{CEDR DFS Database Search Form}

Fill in the entries you know, leave the others blank.

Dataset :
Name:
Description:
Races :
Siseases :
Sites:
Contact-Name:
Subject :
Added-Keywords :
CEDRQAQC:

\section{Submit Query Clear Fileds}




\section{CEDR File Database Browse Form}

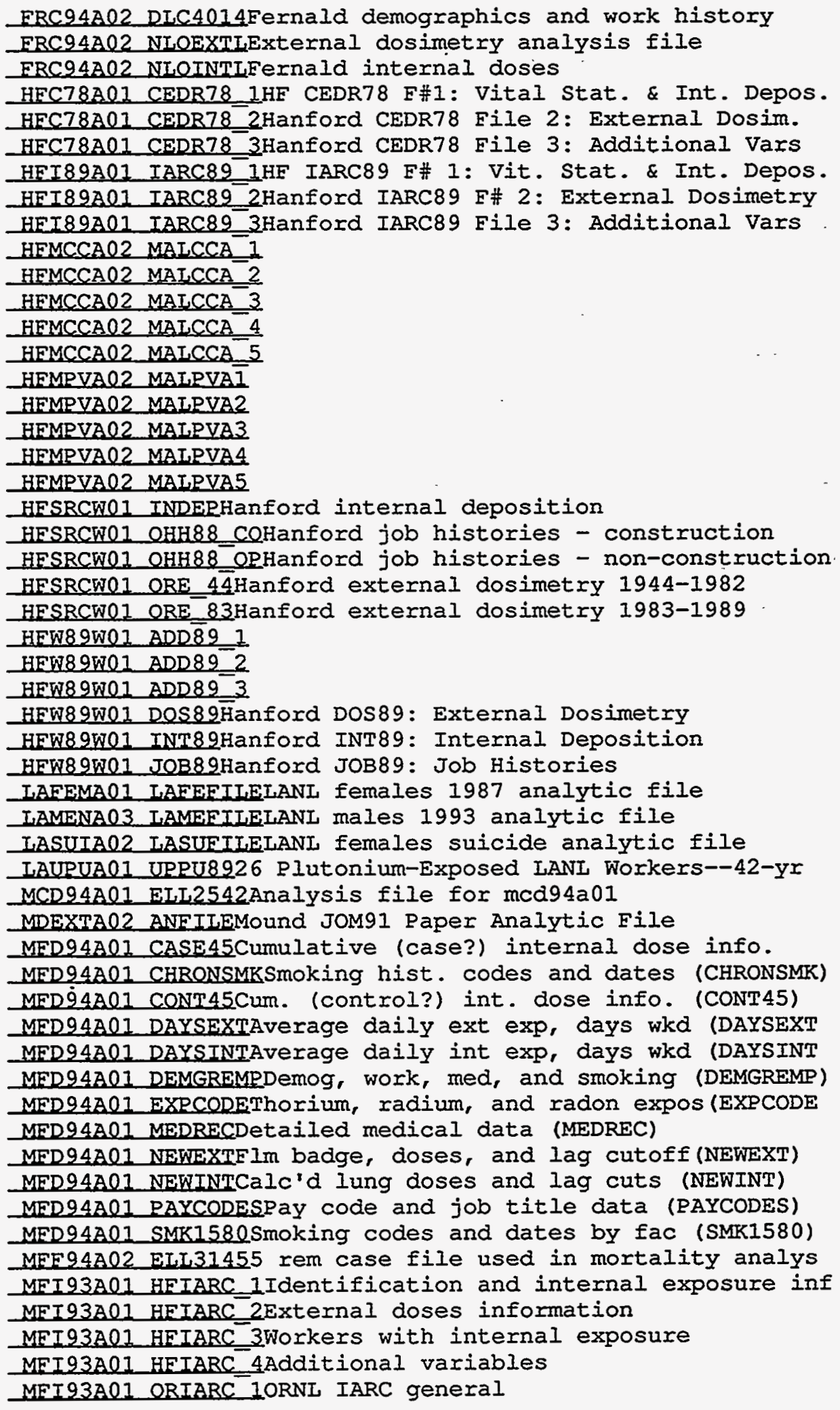




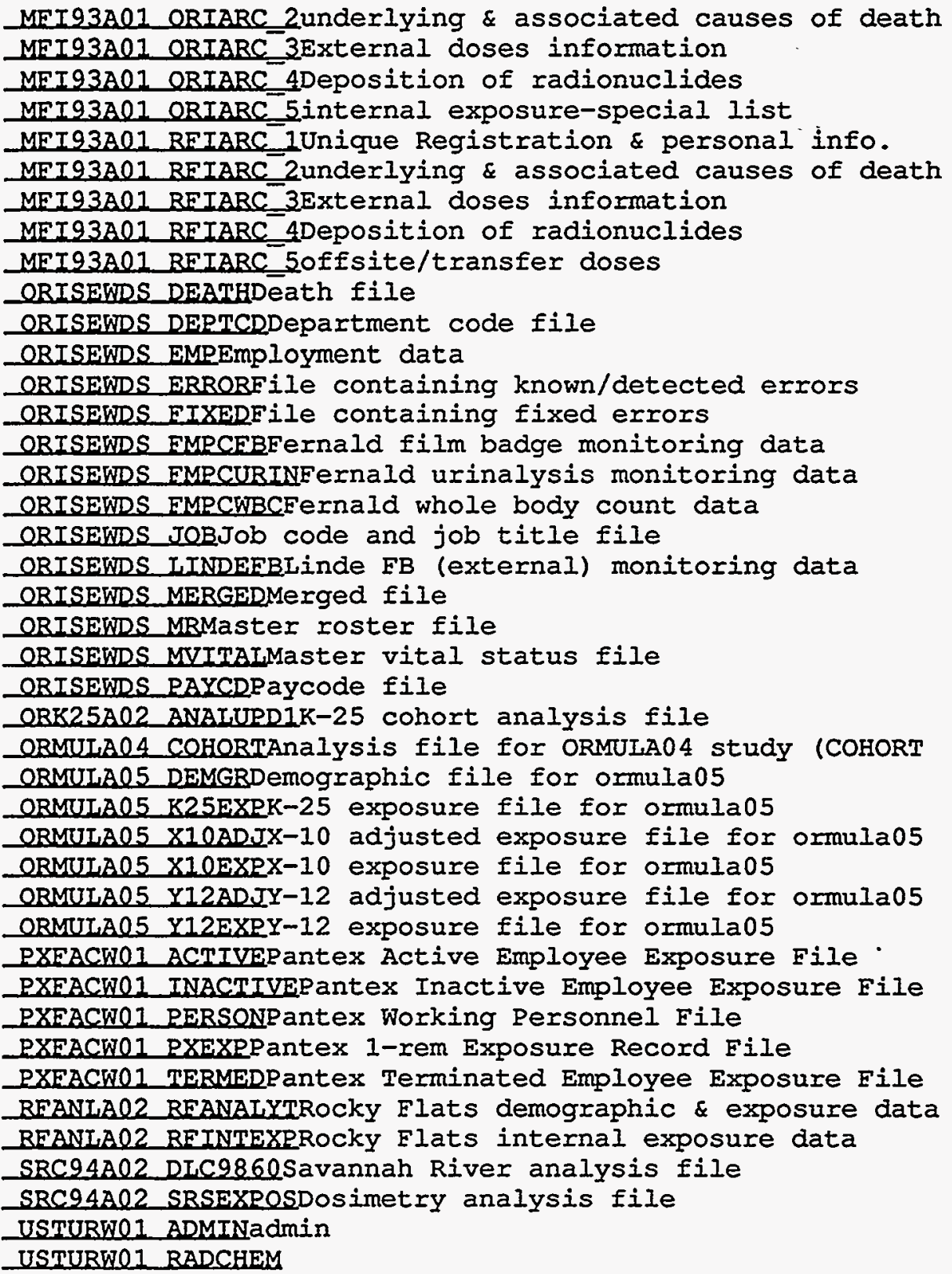




\section{CEDR File Database Search Form}

Fill in the entries you know, leave the others blank.

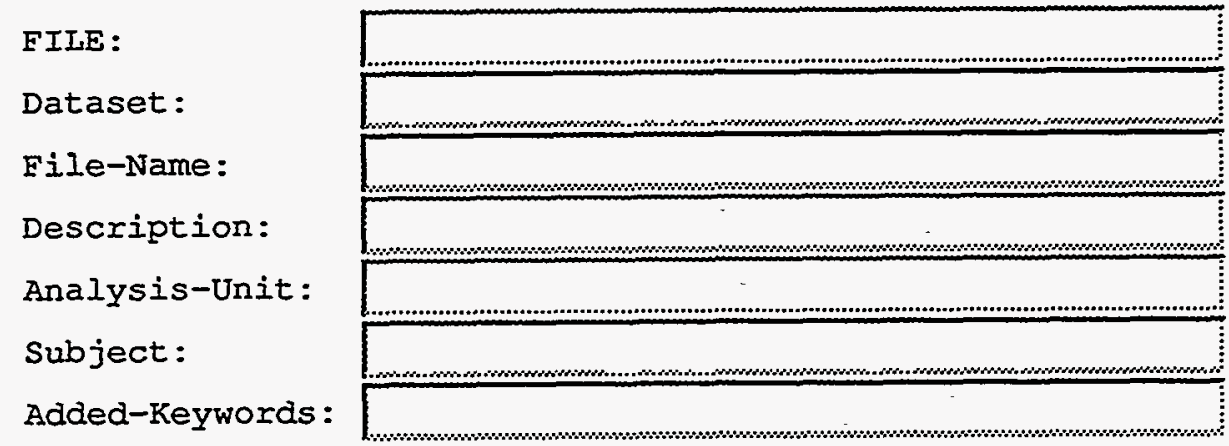

Submit Query Clear Fileds 
Appendix E

Selected Screens from WWW Access to Epidemiology Guides (site archive records) 


\title{
Epidemilogic Guides
}

\section{Prepared for the U.S. Department of Energy \\ Office of Epdemiology and Health Surveillance}

\author{
By \\ History Associates Incorporated \\ Rockville, Maryland
}

\section{Browsing Epidemilogic Guides}

- Hanford Site: A Guide to Record Series Supporting Epidemiologic Studies Coducted for the Department of Energy.

- Los Alamos National Laboratory: A Guide to Records Series Supporting Epidemiologic Studies Condunted for the Department of Energy. [Available Online Soon]

- Oak Ridge Y-12 Plant: A Guide to Records Series Supporting Epidemiologic Studies Conducted for the Department of Energy.

- Oak Ridge K-25 Plant: Records Relating to Cesium at the K-25 Plant: A Guide to Record Series of the Department of Energy and Its Contractors.[Available Online Soon]

- Oak Ridge National Lab and Oak Ridge Operations Office: Records Relating to RaLa, Iodine-131, and Cesium-137 at the ORNL and the Oak Ridge Operations Office

- Oak Ridge Institute for for Science and Education: A Guide to Record Series Supporting Epidemiologic Studies Conducted for the Department of Energy. [Available Online Soon]

- Rocky Flats Fire September 1957: A Guide to Record Series of the Department of Energy and its Contractors.

- Rocky Flats Plant: A Guide to Record Series Useful for Health-Related Research (7 volumes). [Available Online Soon]

\section{Searching in Epidemiolgic Guides}

\section{Search Epiguides}

\section{Searching Epi Data Element Database:}

\section{Search Enide}

Record Series for entire DOE sites

Record Series for DOE sites (not confined to epidemiology) 
This CEDR information retrieval facility developed by Madel Perez, Jose Rivera and Yamilet Rodriguez.

We welcome your comments and questions concerning the CEDR project, our information retrieval products and Customer Service. If you would like further information about CEDR, please contact us at:

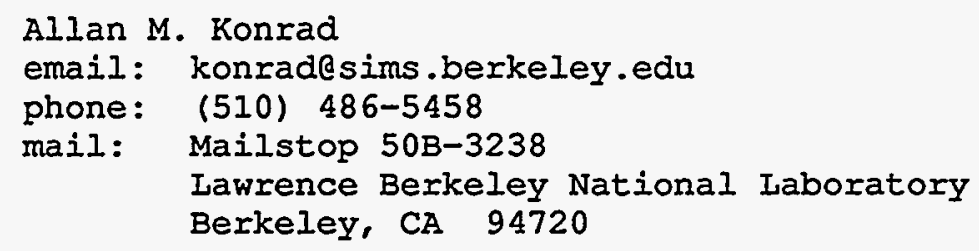

Updated 05 September 1996. 


\section{CEDR Epidguides Database Search Form}

Fill in the entries you know, leave the others blank.

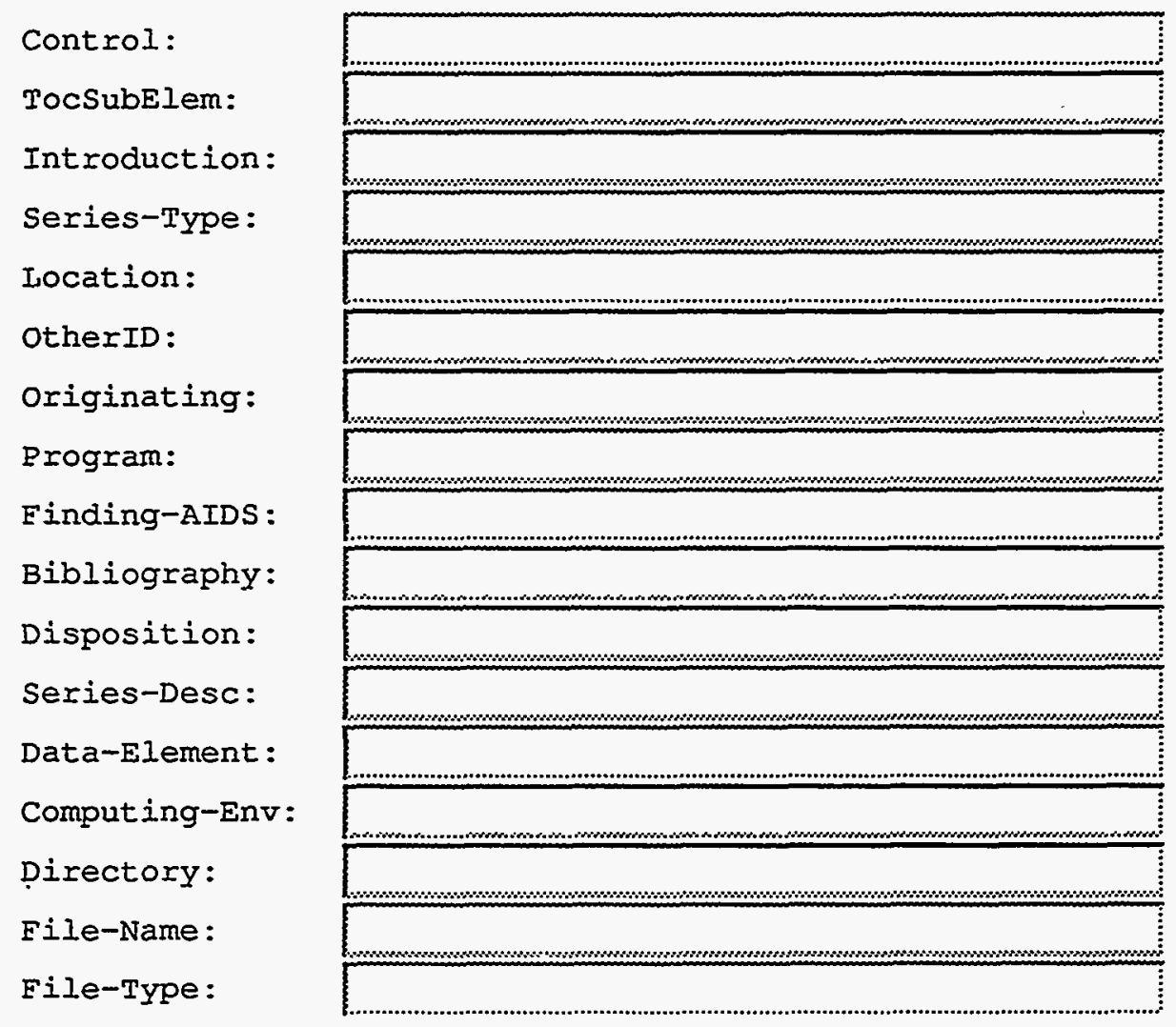

Submit Query Clear Fileds 


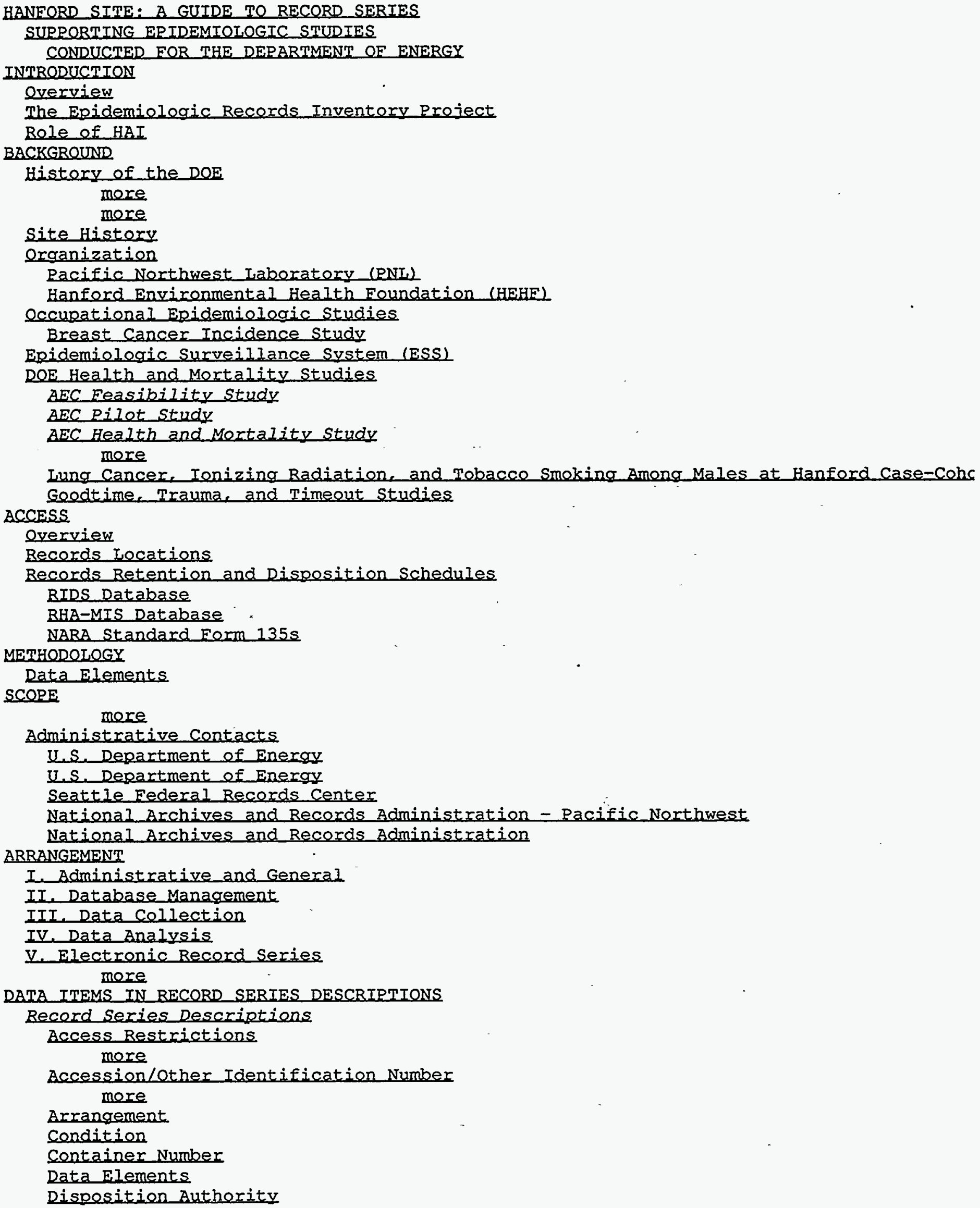


Duplication

Finding Aids

Iocation

Medium

Qriginating office

Scanning suitability

Series Description

Title and Inclusive Dates

Volume

Electronic Record Series Descriptions

Access Restrictions

Data Elements

Directory Name

Disposition Authority

Eile Description

File Name

Eile Tyre

Hardware/Software

Location

office/Program Supported

Originating office

Atomic Energy Commission (AEC) Feasibility Study Preliminary Repert; ca, 1965

Atomic Energy Commission (AEC) Health and Mortality Study (HMS) Progress Reports, $19 f$

Atomic Energy Commission (AEC) Health and Mortality Study (HMS) Repert, 1969

Epidémiologic Study Publications, 1976-1979, 1985, 19871990,1992

Hanford Environmental Health Foundation (HEHF) Annual Reports, 1968-1971

Hanferd Environmental Health Foundation (HEHF) Monthly Reports, 1974-1989

Hanford Exposure Project (HEX) Iog, 1967-1968

Hanford Exposure Project (HEX) Records, ca. 1940-1974

Hanford Health and Mortality Study (HMS) Administrative Files; 1964-1989

Hanford Health and Mortality Study (HMS) Advisory Committee Meeting Records, 1982-198

Industrial Medical Services Section Procedures, $1945 \quad 1946, \quad 1955-1958$

Inspector General Investigation Records, 1977

Joint Epidemiology Group Meeting Minutes, $1984-1990$

Mancuse Correspondence and Study Records, 1965-1978

Occupational Program Records, 1991

Racific Northwest Iaboratory (PNL) General Records, 1979-1992

Pacific Northwest Laboratory (PNL) Monthly Reports, 1987-1994

Skin Cancer Project Study Records, 1987

Timeout Study Records, 1986-1989

Trauma Study Planning Records, 1989

Analysis (ANAI.89) File Edit Records, 1991

center for Epidemiologic Research (CER) Database Cerrelation Records, 1993

Comprehensive Epidemiologic Data Resource (CEDR) Database Correlation Records, 1993

Goodtime Study Database Code Records, 1989

Hanford Health and Mortality Studv (HMS) Database Documentation Records, ca. 1989-19c

Hanferd Mortality (HMO) File Documentation Records, 1979, 1984- 1994

Health Surveillance System (HSS) Development Records, 1986-1988

Health Surveillance System (HSS) Edit Records, 1985-1991

Health Surveillance System (HSS) Ouery Records, 1990-1992

International Agency for Research on cancer. (IARC) Database Correlation Records, 1921

Key File Edit Records, 1992

Master (MST1) File Edit Records, 1987-1994

Menson Fertran Pregram Cede Recerds, 1991

Mertality and Occupational Exposure (MOX) File Documentation Records, 1987

Mertality and Occupational Exposure (MOX) Eile Edit Records, 1987- 1988

Mertality Study Database (MORT) Modification Records, 1991-1993

Qccupational Radiation Exposure (ORE) File Resolution Records, 1983-1989, 1991

Social Security Number Verification Records, 1989-1990, 1992

Software Development Project Materials, 1989-1290

Timeout Study Database Records, 1987-1989 
Benton and Frankin Counties Monthly Mortality Reports, 1988-1993

Benton County Voter Report. 1981

Bloed Type Record Cards, 1964

Breast Cancer Study Internal Exposure Records, 1988-1991

Breast Cancer Study Medical Records, 1984-1989

Building Iists, 1967

Construction Employees: Roster, 1985-1987

Contractor Emplovee Roster Records, 1950-1953

cooperative Program Personnel Ouestionnaires, 1992

Cumulative Radiation Exposure Records, 1969

Death Certificate Records, 1944-1994

External Exposure Data Records, 1983-1989

External Expesure Records, 1969, 1973-1975, 1978, 1982

Goodtime Study Alcohol, Tobacco, and Exercise Data Abstract Forms, 1984-1922 LOCI=RHE

Hanford Area Autopsy Records, undated

Hanford Atomic Power operations (HAPO) Personnel Data, 1264 IOCI=BHA, 712 Building, E

Hanford Employee Cancer Malignancy Mortality Records, undated

Hanford Environmental Health Foundation (HEHE) Death Certificate Retrieval Becords, ]

Health Medical Examination (HME) Report Records, 1944-1976

Health Surveillance System (HSS) Health Event Audit Becerds, 1984-1993

Health Surveillance System (HSS) Health Event Monthly Reports, 1984-1990

Health Surveillance System (HSS) Health Event Records, 1987-1993

Injury and Accident Reports, $1953-1960$

Internal Exposure Records, 1974-1976

Iob Code Records, 1983, 1985

Lung Cancer Study Records, 1983-1994

Master File Records, 1988

Medical Chart Logs, 1966-1967

Mortality Data Validation Repert, 1993

Mortality Record Cards, 1981

Mortality Study Database (MORT) Reports, 1981, 1986-1987, 1992-1993

eccupational Health History Master File Data Records, 1971, 1976, 1978-1986, 1988

occupational Health History Master Files, ca. 1944-1979

Occupational Health Treatment Records, 1978, 1981-1988

operations and Construction Workers Roster Records, undated

Organizational Charts and Directories, 1944-1986

Organizational Code Directory, 1969

Rayroll Records, 1963-1965, 1972, 1974

Rlutonium Internal Deposition Study Records, 1945, 1955-1973, 1981,1983

Public Health and Social Services Recerds, 1963

Social Security Administration (SSA) Emplovee Data Records, 1967-1972

Timeout Study Alcohol, Tobacco, and Exercise Data Abstract Forms, ca. 1989

Trauma Study Alcohol, Tobacco, and Exercise Data Abstract Forms, 1989

Tumor Registry Project Records, 1987

United States Death Rate Records, 1985,1988

$X$-ray Exposure Study Records, 1968, 1982

Health Surveillance System (HSS) Basic Analysis Reports, 1985-1991

Health Surveillance System (HSS) Hanford Roster Analysis, 1287-1992

Leukemia Data Correlation Records, 1992

Medical Department Monthly Reports, 1948-1953

Mortality and Occupational Exposure (MOX) and Monson Reports, 1988-1991, 1923

Rlutonium Finishing Workers Profile Records, 1991

Radiation Expesure Data Records, 1990

Master Eiles

Master Files

Master Files

Master Eiles

Master Files

Master Files

Master Files

Master Files 
Personal Information Files Personal Information Files Personal Information Files Personal Information Files Personal Information Files Personal Information Files Personal Information Files Personal Information Files Work History Files

Work History Files

eccupational Radiation Exposure Files lccupational Radiation Exposure Eiles eccupational Radiation Exposure Files occupational Radiation Exposure Files eccupational Radiation Exposure Files Qccupational Radiation Exposure Files Vital Status Files

Vital Status Files 